\title{
OBJETS COMPACTS DANS LES TOPOS
}

\author{
EDUARDO J. DUBUC and JACQUES PENON
}

(Received 3 April 1984)

Communicated by R. H. Street

\begin{abstract}
It is well known that compact topological spaces are those spaces $K$ for which given any point $x_{0}$ in any topological space $X$, and a neighborhood $H$ of the fibre $\pi^{-1}\left\{x_{0}\right\} \subset K \times X \stackrel{\pi}{\rightarrow} X$, then there exists a neighborhood $U$ of $x_{0}$ such that $\pi^{-1} U \subset H$. If now $K \stackrel{\pi}{\rightarrow} 1$ is an object in an arbitrary topos, in the internal logic of the topos this property means that, for any $A$ in $\Omega$ and $B$ in $\Omega^{K}$, we have $\forall_{\pi}\left(\pi^{-1} A \cup B\right)=A \cup \forall_{\pi} B$. We introduce this formula as a definition of compactness for objects in an arbitrary topos. Then we prove that in the gross topoi of algebraic, analytic, and differential geometry, this property characterizes exactly the complete varieties, the compact (analytic) spaces, and the compact manifolds, respectively.
\end{abstract}

1980 Mathematics subject classification (Amer. Math. Soc.): 18 B 25.

\section{Introduction}

Nous adoptons ici le point de vue intrinsèque dècrit dans [8] où tout objet d'un topos (a priori quelconque) est vu comme un "espace topologique généralisé". Dans cette optique on donne un sens à la phrase " 1 'objet $K$ est compact" en utilisant uniquement la logique du topos. Essentiellement, la propriété usuelle des compacts qui est retenue ici est celle qui nous dit que dans n'importe quel "espace" produit $X \times K$ tout voisinage d'un axe vertical est un voisinage tubulaire. Elle a; en effet, l'aventage d'être valide, non seulement usuellement en topologie, mais aussi en géométrie algébrique, puisqu'elle caractérise, parmi les schémas,

Recherche partiellment subventionnée par le Centre Interuniversitaire en Etudes Catégoriques, Montréal, Québec, Canada.

(c) 1986 Australian Mathematical Society $0263-6115 / 86 \$ A 2.00+0.00$ 
ceux qui sont complets (du moins quand on se limite au cas algébriquement clos). Cette propriété, exprimé dans la logique du topos prend la forme suivante:

$$
\forall F \subset K \times X \forall x \in X \quad \frac{\neg \pi^{-1}\{x\} \cup F=X \times K}{\neg\{x\} \cup \forall_{\pi} F=X}
$$

où $\pi$ est la projection $X \times K \rightarrow X$. Le voisinage tubulaire est donc $\pi^{-1} \forall_{\pi} F \subset F$. Signalons enfin que tous les modèles envisagés ici sont des "gros" topos de la topologie, de la géométrie algébrique et les modèles bien adaptés à la géométrie différentielle.

\section{Considérations générales}

\section{Ouverts et séparés dans un topos}

Dans tout ce chapitre $\mathscr{E}$ désignera un tops fixé. Aussi lorsqu'on parlera d'objects ou flèches sera-t-il sous-entendu qu'ils appartiennent à $\mathscr{E}$. Rappellons [8] la définition suivante.

1. Definition [8]. Etant donné un object $X$, un sous-object $U$ de $X$ est appellé ouvert s'il satisfait le formules

$$
\forall x_{0}\left(x_{0} \in U \rightarrow \forall x\left(x \neq x_{0} \vee x \in U\right)\right) .
$$

Intuitivement cela signifie que tout point de $X$ est soit "loin de $x_{0}$ " (que l'on écrit $x \neq x_{0}$ ) soit dans $U$. Ou encore, que tout point "suffisamment prés de $x_{0}$ " est dans $U$. C'est une autre façon de dire que $U$ est "voisinage" de chacun de ses points.

2. Proposition [8] (Propriétés des ouverts). Les ouverts sont stables

(1) par intersections finis,

(2) réunion queconque (interne),

(3) changement de base (i.e. toute flèche est "continue"), et

(4) composition.

3. Definition-Proposition. Un objet $X$ est appellé séparé s'il satisfait une des formules équivalentes suivantes:

(i) $\forall x \forall y \forall z(x \neq y \rightarrow z \neq x \vee z \neq y)$;

(ii) $\forall x(\neg\{x\}$ ouvert de $X)$;

(iii) $\neg(\Delta \nrightarrow X \times X)$ est ouvert. 
Preuve. (i) $\Rightarrow$ (iii). Soient $x, y, x^{\prime}$ et $y^{\prime}$ des éléments de $X$ alors, comme $X$ est séparé on a

$$
\begin{array}{ll} 
& x \neq y \rightarrow x \neq y^{\prime} \vee y \neq y^{\prime}, \\
& x \neq y^{\prime} \rightarrow x \neq x^{\prime} \vee y^{\prime} \neq x^{\prime}, \\
\text { donc } & x \neq y \rightarrow x \neq x^{\prime} \vee x^{\prime} \neq y^{\prime} \vee y \neq y^{\prime}, \\
\text { deplus } & x \neq x^{\prime} \vee y \neq y^{\prime} \rightarrow \neg\left(x=x^{\prime} \vee y=y^{\prime}\right), \\
\text { par suite } & x \neq y \rightarrow \neg\left(x=x^{\prime} \wedge y=y^{\prime}\right) \vee x^{\prime} \neq y^{\prime}, \\
\text { où encore } & (x, y) \in \neg \Delta \rightarrow(x, y) \neq\left(x^{\prime}, y^{\prime}\right) \vee\left(x^{\prime}, y^{\prime}\right) \in \neg \Delta,
\end{array}
$$

où $\Delta$ désigne la diagonale dans $X \times X$.

(iii) $\Rightarrow$ (ii). Les ouverts étant stables par changement de base, il suffit de considérer l'application $y \rightarrow(x, y)$ de $X$ dans $X^{2}$.

(ii) $\Rightarrow$ (i). C'est évident.

4. Proposition. Soient $X$ et $Y$ deux objets tels que $X \times Y$ est séparé, alors

$$
\forall x \forall x^{\prime} \forall y \forall y^{\prime}\left[\neg\left(x=x^{\prime} \wedge y=y^{\prime}\right) \rightarrow \neg\left(x=x^{\prime}\right) \vee \neg\left(y=y^{\prime}\right)\right] .
$$

Preuve. L'hypothèse $\left[\neg\left(x=x^{\prime} \wedge y=y^{\prime}\right)\right]$ peut encore s'écrire: $\neg[(x, y)=$ $\left.\left(x^{\prime}, y^{\prime}\right)\right]$ par suite, comme $X \times Y$ est sépare, on a

$$
\neg\left[\left(x, y^{\prime}\right)=(x, y)\right] \vee \neg\left[\left(x, y^{\prime}\right)=\left(x^{\prime}, y^{\prime}\right)\right]
$$

d'où la conclusion de la proposition.

5. Remarque. Kock donné dans [5] une définition de corps sensiblement plus forte que celle de corps de fraction. Grace à la Proposition 4 on voit que un corps de fraction $K$ est un "corps au sens de Kock" si et seulement si $K^{n}$ est séparé pour tout entier $n$.

\section{Objets compacts dans un topos}

6. Definition. Un objet $K$ est appellé compact s'il satisfait la formule

(C1) $\forall A \in \Omega \forall B \in \Omega^{K}[\forall k \in K(A \vee B(k)) \rightarrow A \vee \forall k \in K B(k)]$.

Exprimé plus globalement, la formule entre crochets peut s'écrire

$$
\forall_{\pi}\left(\pi^{-1} A \cup B\right) \subset A \cup \forall_{\pi} B,
$$

où encore,

$$
\frac{\pi^{-1} A \cup B=K}{A \cup \forall_{\pi} B=1}
$$


REMARQue. Dans le cas particulier où $A$ est de la forme $\neg_{C}$ la formule entre crochets peut encore s'écrire sous la forme d'une adjonction:

$$
\frac{\pi^{-1} C \ll B}{C \ll \forall_{\pi} B}
$$

où la relation $P \ll Q$ signifie $\neg P \cup Q=X$ si $P$ et $Q$ sont des sous-objets de $X$.

$K$ étant un compact et $X$ un objet quelconque, on vérifie immédiatement que $(\mathrm{C} 1, \mathrm{X})$

$\forall A \in \Omega^{X} \forall B \in \Omega^{K \times X}[\forall x \forall k(A(x) \vee B(k, x)) \rightarrow \forall x(A(x) \vee \forall k B(k, x))]$ que l'on peut encore écrire

$$
\frac{\pi^{-1} A \cup B=K \times X}{A \cup \forall_{n} B=X}
$$

où $\pi: K \times X \rightarrow X$ désigne la projection canonique.

Enfin considérons aussi le cas particulier à $A=\neg\left\{x_{0}\right\}$. On a donc $(\mathrm{C} 2, \mathrm{X})$

$\forall x_{0} \in X \forall B \in \Omega^{K \times X}\left[\forall x \forall k\left(x \neq x_{0} \vee B(k, x)\right) \rightarrow \forall x\left(x \neq x_{0} \vee \forall k B(k, x)\right)\right]$ que l'on peut encore écrire

$$
\frac{\neg \pi^{-1}\left\{x_{0}\right\} \cup B=K \times X}{\neg\left\{x_{0}\right\} \cup \forall_{\pi} B=X} .
$$

Exprimé intuitivement, cette propriété signifie que tout vorsinage d'une axe est un voisinage tubulaire (le tube étant $\pi^{-1} \forall_{\pi} B$ )

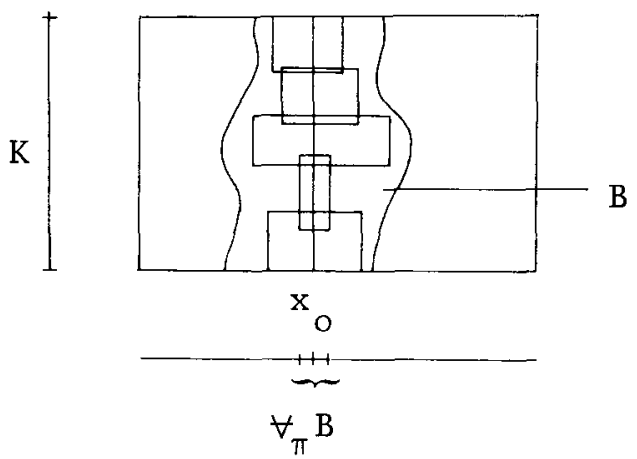

C'est clairement une propriété des espaces compacts. Nous pouvons recouvrir l'axe par des pavés auverts $U_{\alpha} \times V_{\alpha}$. Comme la famille $\left(U_{\alpha}\right)$ recouvre $K$ on peut en extraire un sous-recouvrement fini $\left(U_{\alpha}\right)_{\alpha \in F}$. Il suffit alors de considérer $V=\bigcap_{\alpha \in F} V_{\alpha}$ pour obtenir le voisinage tubulaire $K \times V$ cherché.

Une autre propriété classique de la compacité est que les projections $K \times X \rightarrow X$ sont des applications fermées, ce que l'on peut exprimer en disant que l'application $\forall_{\pi}$ préserve les ouverts (en remarquant que $\forall_{\pi}=\neg \exists_{\pi} \neg$, due moins dans 
$\mathscr{E}$ ns!) C'est-à-dire

(C3, X)

$$
\forall U \in \Omega^{K \times X} \text { ( } U \text { ouvert } \rightarrow \forall_{\pi} U \text { ouvert) } .
$$

La proposition suivante compare (lorsque le topos $\mathscr{E}$ est quelconque) les différantes propriétés $(\mathrm{C} 1),(\mathrm{C} 1, \mathrm{X}),(\mathrm{C} 2, \mathrm{X}),(\mathrm{C} 3, \mathrm{X})$.

7. Proposition. (1) (C1) $\Leftrightarrow$ pour tout $X,(\mathrm{C} 1, \mathrm{X})$,

(2) $(\mathrm{C} 1, \mathrm{X}) \Leftrightarrow(\mathrm{C} 2, \mathrm{X})$, pour tout $X$,

(3) $(\mathrm{C} 2, \mathrm{X}) \Rightarrow(\mathrm{C} 3, \mathrm{X})$, pour tout $X$ tel que $K \times X$ est séparé.

Preuve. (1) et (2) étant claires, montrons simplement (3). Soit $x \in \forall_{\pi} U$, alors,

$$
\begin{gathered}
\frac{x \in \forall_{\pi} U}{\frac{\forall k[(k, x) \in U]}{\left.\left(k=k^{\prime} \wedge x=x^{\prime}\right) \vee\left(k^{\prime}, x^{\prime}\right) \in U\right]}} \\
\frac{\forall k \forall k^{\prime} \forall x^{\prime}\left[\neg \left(k k^{\prime} \forall x^{\prime}\left[\neg\left(x=x^{\prime}\right) \vee \neg\left(k=k^{\prime}\right) \vee\left(k^{\prime}, x^{\prime}\right) \in U\right]\right.\right.}{\forall x^{\prime}\left[x \neq x^{\prime} \vee \forall k \forall k^{\prime}\left(k \neq k^{\prime} \vee\left(k^{\prime}, x^{\prime}\right) \in U\right)\right]}
\end{gathered}
$$

en particulier lorsque $k=k^{\prime}$ on a

ce qui prouve que $\forall_{\pi} U$ est ouvert.

$$
\frac{\forall x^{\prime}\left[x \neq x^{\prime} \vee \forall k\left(\left(k, x^{\prime}\right) \in U\right)\right]}{\forall x^{\prime}\left[x \neq x^{\prime} \vee x^{\prime} \in \forall_{\pi} U\right]}
$$

L'équivalence entre toutes les propriètés discutés précédement est probablement fausse, en toute généralité.

Nous allons voir (cf. "Contexte II") que, dans de nombreaux exemples on a pourtant l'implication: [Pour tout $X$ séparé, $(\mathrm{C} 3, \mathrm{X})] \Rightarrow(\mathrm{C} 1)$.

\section{Contextes particulieres}

\section{Ouverts et séparés}

Les Contextes I et II que nous allons présenter ici ont été donnés dans [9] c'est pourquoi ne nombreuses propositions de ce paragraphe seront signalées sans démonstration. Ce sont elles qui permettront au Chapitre III de caractériser les séparés et compacts dans les exemples ainsi que de répondre au problème posé à la fin du Chapitre I.

\section{CONTEXTE I}

Soit $\mathscr{C}$ une catégorie a limites projective finies et objet initial 0 , avec $0 \neq 1$. vérifiant le "Nullestellensatz", c'est-à-dire

(0) pour tout $X$ de $\mathscr{C}, \Gamma X=\varnothing$ si et seulement si $X=\varnothing$, où $\Gamma=\operatorname{Hom}(1,-)$. 
Considérons, deplus, une topologie sur $\mathscr{C}$ vérifiant les propriétés suivantes.

(1) Elle est sous-canonique (i.e. tout préfaisceau sur $\mathscr{C}$, représentable, est un faisceau).

(2) $\mathrm{Si}\left(X_{i} \rightarrow X\right)$ couvre, alors $\left(\Gamma X_{i} \rightarrow \Gamma X\right)$ est surjective,

(3) La famille vide $(\rightarrow 0)$ couvre 0 .

Soit $\mathscr{E}$ le topos des faisceau sur $\mathscr{C}$. On vérifie facilement que $\mathscr{E}$ satisfait aussi le "Nullestellensatz".

8. Proposition [9]. Soit $X$ un objet de $\mathscr{E}$ et $S$ une partie de $\Gamma X$, alors il existe un sous-objet $E(X, S)$ de $X$ tel que
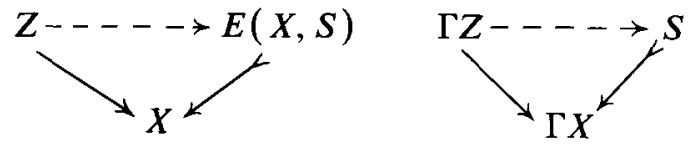

pour tout $Z \rightarrow X$ dans $\mathscr{E}$.

En particulier, l'application $E(X,-)$ est un fontcteur $P(\Gamma X) \rightarrow P(X)$ adjoint à droite du foncteur $\Gamma P(X) \rightarrow P(\Gamma X)$, où $P(-)$ désigne le treillis (externe) des sous-objets.

Par la suite, s'il n' y a pas d'ambiguté nous écrirons simplement, par abus de notation, $E S$ au lieu de $E(X, S)$.

On vérifie facilement que $\Gamma E=$ Id.

Nous allons maintenant étudier comment $\Gamma$ et $E$ transporten les logiques respectives de $\mathscr{E}$ et $\mathscr{E}$ ns. Pour cela, nous utiliserons les mêmes notations pour désigner les symboles logiques des deux topoi. Aussi, lorsque $f: Y \rightarrow X$ est une flèche de $\mathscr{E}$, noterons nous, de la même façon, la flèche correspondante $\Gamma Y \rightarrow \Gamma X$.

9. Proposition. Soit $f: Y \rightarrow X$ une flèche de $\mathscr{E}$. Considérons le diagramme suivant:

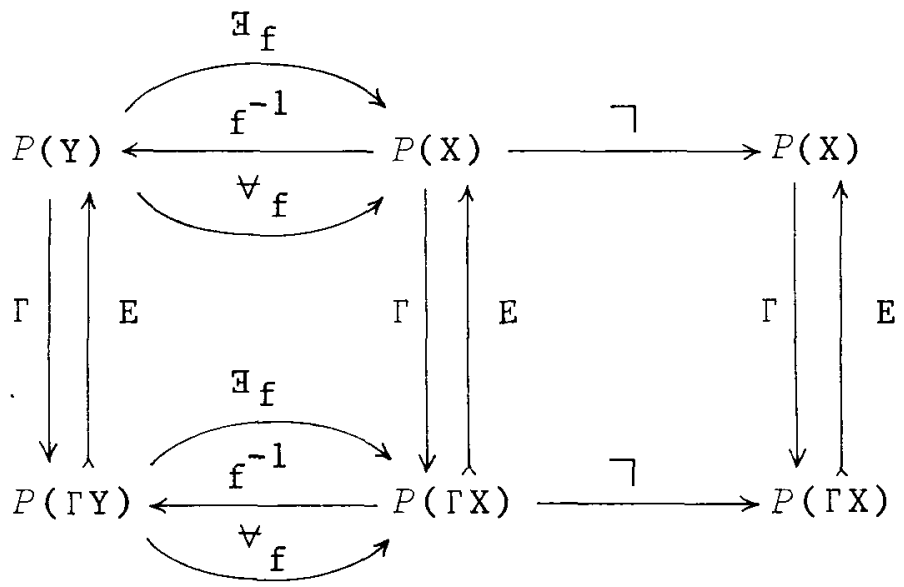


Alors $\exists_{f} \dashv f^{-1} \dashv \forall_{f}, \Gamma \dashv E . E t$, par composition d'adjoints

$$
\begin{array}{ll}
\Gamma \exists_{f} \dashv f^{-1} E & f^{-1} \Gamma \dashv \exists \forall_{f} \\
\exists_{f} \Gamma-E f^{-1} & \Gamma f^{-1} \dashv \forall_{f} E
\end{array}
$$

Nous avons deplus les équations suivantes:
(1) $E \neg=\neg E$
(7) $\Gamma \neg=\neg \Gamma$
(2) $E f^{-1}=f^{-1} E$
(8) $\Gamma f^{-1}=f^{-1} \Gamma$
(3) $E \forall_{f}=\forall_{f} E$
(9) $\Gamma \forall_{f} E=\forall_{f} \Gamma E$
(4) $\Gamma E \exists_{f}=\Gamma \forall_{f} E$
(10) $\Gamma \exists_{f}=\exists f \Gamma$
(5) $\Gamma E(-\cup-)=\Gamma(E(-) \cup E(-))$
(11) $\Gamma\left(-\cup_{-}\right)=\Gamma(-) \cup \Gamma(-)$
(6) $\Gamma(-\cap-)=E(-) \cap E(-)$
(12) $\Gamma(-\cap-)=\Gamma(-) \cap \Gamma(-)$.

Preuve. On vérifie facilement (2) et (6) car $E$ a un adjoint à gauche; (11) car $\Gamma$ a un adjoint à droite; (8) et (12) car $\Gamma$ est représentable; (3) et (10) résultent de (8) et (2) par composition d'adjonctions; (4), (5) et (9) résultent de (10), (11) et (3) car $\Gamma E=$ Id

Il reste à montrer (1) et (7). Comme $\Gamma E=I d,(7)$ se déduit de la propriété ( $\left.7^{\prime}\right)$ $\neg=E \neg \Gamma$. Montrons donc (1) et $\left(7^{\prime}\right)$ :

(1)

$$
\frac{\frac{Q \subset \neg E S}{Q \cap E S=0}}{\frac{\Gamma(Q \cap E S)=\varnothing}{\Gamma Q \cap \Gamma E S=\varnothing}} \frac{\Gamma Q \cap S=\varnothing}{\frac{\Gamma Q \subset S}{Q \subset E \neg S}}
$$

$$
\frac{\frac{P \subset \neg Q}{P \cap Q \subset 0}}{\frac{\Gamma(P \cap Q) \subset \varnothing}{\Gamma P \cap \Gamma Q \subset \varnothing}} \frac{\Gamma P \subset \neg \Gamma Q}{P \subset E_{\neg} \Gamma Q}
$$

CONTEXTE II

Soit $\mathscr{C}$ une catégorie à limites projectives finies et objet initial 0 , avec $1 \neq 0$. Comme précédamment écrivons $\Gamma$ pour le foncteur section globale. Soit deplus $\mathscr{U}$ une classe de monomorphismes de $\mathscr{C}$ telle que

(1) $\mathscr{U}$ est stable par changement de base,

(2) $\mathscr{U}$ est stable par composition,

(3) Pour toute famille $\left(U_{i} \nrightarrow X\right)$ de $\mathscr{U}$, si $\left(\Gamma U_{i} \nrightarrow \Gamma X\right)$ est surjective alors $\left(U_{i} \mapsto X\right)$ est épimorphe effective.

10. Proposition [9]. Contexte II $\Rightarrow$ Contexte I (en prennant pour topologie sur $\mathscr{C}$ les familles $\left(U_{i} \mapsto X\right)$ de $\mathscr{U}$ telles que $\left(\Gamma U_{i} \rightarrow \Gamma X\right)$ est surjective $)$. 
Soit $\mathscr{E}$ le topos des faisceaux sur $\mathscr{C}$.

$X$ étant un object de $\mathscr{E}$ on va munir $\Gamma X$ d'une structure d'espace topologique notée Spec $X$.

(1) Lorsque $X$ est représentable, Spec $X$ a pour base d'ouverts la famille des sous-ensembles de la forme $\Gamma U \hookrightarrow \Gamma X$, lorsque $U$ parcoure $\mathscr{U}$.

(2) Si $X$ est quelconque dans $\mathscr{E}$, Spec $X$ est la topologie finale déterminée par les familles d'applications (Spec $Z \stackrel{\Gamma f}{\rightarrow} \Gamma X$ ) où $Z$ parcoure les objets représentables de $\mathscr{E}$.

Il est claire que si $f: Y \rightarrow X$ est une fleche de $\mathscr{E}$, Spec $Y \rightarrow \operatorname{Spec} X$ est continue.

11. Proposition [9]. $X$ étant un objet de $\mathscr{E}$ et $Q \subset X$, alors

$$
Q \text { ouvert dans } X \Rightarrow \Gamma Q \text { ouvert dans } \operatorname{Spec} X \text {, et } Q=E \Gamma Q \text {. }
$$

12. Corollaire. Pour les sous-objets ouverts $U$ de $\mathscr{E}$ on a les équations

$$
\Gamma \forall_{f}(U)=\forall_{F} \Gamma(U)
$$

13. Definition [9]. On dira qu'un objet $X$ de $\mathscr{E}$ est Haussdorf si la diagonale $\Gamma X \nrightarrow \operatorname{Spec}(X \times X)$ est un sous-ensemble fermé.

Remarque. Notons que Spec $X$ n'est pas nécéssairement Haussdorf car le foncteur Spec ne préserve pas, en général, les produits.

14. Proposition [9] (caractérisation des ouverts). Soit $X$ un objet Haussdorf dans $\mathscr{E}, Q \leadsto X$ et $S \nrightarrow$ Spec $X$ des sous-objets, alors

(i) $Q$ ouvert de $X \Leftrightarrow \Gamma Q$ ouvert de $\operatorname{Spec} X$,

(ii) $S$ ouvert de Spec $X \Leftrightarrow E S$ ouvert de $X$.

15. Corollaire. Soit $f: Y \rightarrow X$ une flèche de $\mathscr{E}$, où $Y$ est Haussdorf. Alors les deux propriétés suivantes sont équivalentes

(1) $\exists_{f}: P(Y) \rightarrow P(X)$ préserve les sous-objets ouverts,

(2) $\operatorname{Spec}(Y) \rightarrow \operatorname{Spec}(X)$ est une application ouverte.

Preuve. $(1) \Rightarrow(2)$ car, $U$ étant un ouvert de $\operatorname{Spec}(Y), \exists_{f}(U)=\Gamma E \exists_{f}(U)=$ $\Gamma \exists_{f} E(U)$ (d'aprés la Proposition 9, Equation (4)), (2) $\Rightarrow(1)$ résulte de l'Equation (10) de la Proposition 9.

Remarque. En particulier, lorsque $X \times Y$ est Haussdorf, Spec $\pi$ est ouverte, où $\pi: X \times Y \rightarrow X$ désigne la projection canonique (on utilise la Proposition 2). 
Soit maintenant $f: Y \rightarrow X$ une flèche de $\mathscr{E}$ "ouverte" dans le sens du Corollaire 15 , alors

16. Corollaire. Si $X$ et $Y$ sont Haussdorf, alors pour les sous-objets ouverts, les équations (4), (5) et (9) de la Proposition 9 sont satisfaites "au sens fort". $C$ 'est-à-dire, si $U$ et $U^{\prime}$ sont des ouverts de Spec $Y$ et $V$ un sous-objet ouvert de $Y$, alors

$$
\begin{gathered}
E \exists_{f}(U)=\exists_{f} E(U) \\
E\left(U \cup U^{\prime}\right)=E(U) \cup E\left(U^{\prime}\right) \\
\Gamma \forall_{f}(V)=\forall_{f} \Gamma(V) .
\end{gathered}
$$

Preuve. Il résulte du corollaire précédant et des Propositions 9 et 14.

Ainsi, pour les sous-objets ouverts, toute la logique de $\mathscr{E}$, "coincide" avec celle de $\mathscr{E}$ ns via $\Gamma$ et $E$. Elle est donc "classique".

17. Proposition. Les objets Haussdorfs sont stables par produits finis dans $\mathscr{E}$.

Preuve. Découle du fait que l'identité $\operatorname{Spec}[(X \times X) \times(Y \times Y)] \rightarrow \operatorname{Spec}(X \times$ $X) \times \operatorname{Spec}(Y \times Y)$ est continue, pour tout $X$ et $Y$.

18. Proposition. Dans $\mathscr{E}$, séparé $\Leftrightarrow$ Haussdorf.

Preuve. Soit $X$ un objet de $\mathscr{E}$.

Si $X$ est séparé, $\neg \Delta$ est ouvert dans $X \times X$, donc $\Gamma \neg \Delta$ est ouvert dans Spec $(X \times X)$ (d'aprés la Proposition 11). Mais $\Gamma \neg \Delta=\neg \Gamma \Delta$ (Proposition 9, Equation (1)), et $\Gamma \Delta=\Delta$. Donc $X$ est Haussdorf.

Réciproquement, si $X$ est Haussdorf, on peut, cette fois, appliquer la Proposition 14 (car $X \times X$ est Hausdorff par la Proposition 17). Elle entraine alors immédiatement que $X$ est séparé.

En combinant les résultats des deux dernières propositions on constate que dans $\mathscr{E}$ les objets séparés sont stables par produits finis, ce qui ne semble pas être vrai dans le cas général.

\section{Objets compacts}

Reprennon, maintenant d'étude comparative des différentes conditions (C1), $(\mathrm{C} 1, \mathrm{X}),(\mathrm{C} 2, \mathrm{X}),(\mathrm{C} 3, \mathrm{X})$. Pour cela, considérons ici des versions "externes" de $(\mathrm{C} 1, \mathrm{X})$ et $(\mathrm{C} 3, \mathrm{X})$ :

(exC1, X) Pour tous sous-objets $A \subset X$ et $B \subset K \times X$,

$$
\frac{\pi^{-1} A \cup B=K \times X}{A \cup \forall_{\pi} B=X}
$$


(exC3, X) Pour tout sous-objet $U \subset K \times X$,

$$
U \text { ouvert } \Rightarrow \forall_{\pi} U \text { ouvert. }
$$

19. Proposition. Soient $K$ et $X$ deux objets de $\mathscr{E}$ tels que $K$ satisfait $(\mathrm{exC} 3, \mathrm{X})$, alors pour tous-objets ouverts $U_{1}$ et $U_{2}$ de $K \times X$, on a

$$
\frac{U_{1} \cup U_{2}=K \times X}{\exists_{\pi} U_{1} \cup \forall_{\pi} U_{2}=X} .
$$

Preuve. On a successivement

$$
\begin{array}{ll}
\frac{U_{1} \cup U_{2}=K \times X}{\Gamma U_{1} \cup \Gamma U_{2}=\Gamma K \times \Gamma X} & \begin{array}{l}
\text { Equation (11) } \\
\text { logique "classique" }
\end{array} \\
\frac{\exists_{\pi} \Gamma U_{1} \cup \forall_{\pi} \Gamma U_{2}=\Gamma X}{\Gamma \exists_{\pi} U_{1} \cup \Gamma \forall_{\pi} U_{2}=\Gamma X} & \text { Equation (10) et Corollaire } 12 \\
\frac{\Gamma\left(\exists_{\pi} U_{1} \cup \forall_{\pi} U_{2}\right)=\Gamma X}{\exists_{\pi} U_{1} \cup \forall_{\pi} U_{2}=X} & \text { Equation (11) }
\end{array}
$$

Cette dernière ligne est justifiée car $\exists_{\pi} U_{1} \cup \forall_{\pi} U_{2}$ est un ouvert (Proposition 2). It suffit alors d'appliquer la Proposition 11.

20. Proposition. Soient $X$ un objet représentable et séparé dans $\mathscr{E}$, et $P$ et $Q$ deux sous-objets de $X$. Alors, si $P$ et $Q$ recouvrent $X$ (i.e. $P \cup Q=X)$ il existe deux ouverts $U_{1}$ et $U_{2}$ de $X$, respectivement contenus dans $P$ et $Q$, qui recouvrent $X$, (i.e. $U_{1} \subset P, U_{2} \subset Q$ et $U_{1} \cup U_{2}=X$ ).

Preuve. $X$ étant représentable, il existe une famille couvrante $\left(U_{i} \nrightarrow X\right)$ telle que $U_{i} \subset P$ ou $U_{i} \subset Q$. Soit $U_{1}$ (respectivement $U_{2}$ ) $=U_{i} U_{i}$ pour les indices $i$ tels que $U_{i} \subset P$ (respectivement $U_{i} \subset Q$ ). Alors $U_{1} \cup U_{2}=X$. $U_{1} \subset P$ et $U_{2} \subset Q$. Deplus, comme chacun des $U_{i}$ est ouvert (car $U_{i}$ est ouvert par définition, et $X$ est séparé) il en est de même de leurs réunions $U_{1}$ et $U_{2}$.

Par la suite, on conviendra d'appeller localement représentable tout objet $X$ recouvert par des ouverts représentables (i.e. il existe une famille $\left(U_{i} \nrightarrow X\right)$ de sous-objets ouverts telle que $X=\bigcup_{i} U_{i}$ et où chaque $U_{i}$ est représentable).

Remarquons que cette situation correspond, dans le cas de la géométrie algébrique, à la définition des schémas.

21. Corollaire. Soit $X$ un objet de E̊ localement représentable par des objets séparés. Alors la conclusion de la Proposition 20 est encore valide.

Preuve. C'est un exercice facile. 
22. Proposition. $K$ étant un objet séparé dans $\mathscr{E},(\mathrm{C} 2, \mathrm{X}) \Rightarrow(\mathrm{C} 3, \mathrm{X})$ pour tout objet $X$ séparé.

Preuve. Les objets séparés étant stables par produits finis (voir remarque aprés la Proposition 18), il suffit d'appliquer la Proposition 7.

23. Proposition. Si $K$ est un objet localement représentable par des séparés, alors $(\mathrm{exC} 3, \mathrm{X}) \Rightarrow(\mathrm{ex} \mathrm{C} 1, \mathrm{X})$ pour tout $\mathrm{X}$ séparé et représentable.

Preuve. Soient $P \subset K \times X$ et $Q \subset X$ tels que $\pi^{-1} Q \cup P=K \times X$; alors, comme $K \times X$ satisfait les conditions du Corollaire 21 (car les ouverts sont stables par changement de base voir Proposition 2) il existe des ouverts $U_{1}$ et $U_{2}$ de $K \times X$ tels que $U_{1} \cup U_{2}=K \times X, U_{1} \subset \pi^{-1} Q$, et $U_{2} \subset P$. Appliquons maintenant la Proposition 19. On a donc $\exists_{n} U_{1} \cup \forall_{\pi} U_{2}=X$. Mais $\exists_{\pi} U_{1} \subset Q$, et $\forall_{\pi} U_{2} \subset$ $\forall_{\pi} P$. Par suite $Q \cup \forall_{\pi} P=X$.

La théorie générale des topos nous enseigne aussi que

24. Proposition. Si, pour tout X représentable, on a (exC1, X), alors on a (C1).

En résumé, nous avons montré que

(1) (pour tout $X$ représentable $(\mathrm{exC} 1, \mathrm{X})) \Rightarrow(\mathrm{C} 1)$,

(2) $(\mathrm{C} 1) \Rightarrow$ (pour tout $X,(\mathrm{C} 1, \mathrm{X})$ ),

(3) $(\mathrm{C} 1, \mathrm{X}) \Rightarrow(\mathrm{C} 2, \mathrm{X})$,

(4) $(\mathrm{C} 2, \mathrm{X}) \Rightarrow(\mathrm{exC} 3, \mathrm{X})$.

Ces trois implications étant vrai en toute généralité et sans restriction sur $K$ et $X$.

(5) $(\mathrm{C} 2, \mathrm{X}) \Rightarrow(\mathrm{C} 3, \mathrm{X})$

dans le Contexte II lorsque $K$ et $X$ sont séparés (i.e. Haussdorf).

(6) $(\mathrm{exC} 3, \mathrm{X}) \Rightarrow(\mathrm{exC} 1, \mathrm{X})$

dans le Contexte II lorsque $K$ est "localement représentable et séparé" (voir la Proposition 23) et $X$ est séparé.

Nous povons donc énoncer le théorème suivant.

25. ThEORÈmE I. Soit $\mathscr{E}$ un topos vérifiant les conditions du Contexte II, et tel que tout objet représentable soit séparé. Alors, pour un objet $K$ séparé et localement représentable, les conditions suivantes sont équivalentes:

(i) $(\mathrm{exC} 1, \mathrm{X})$, pour tout $\mathrm{X}$ représentables;

(ii) $(\mathrm{C} 1)$;

(iii) $(\mathrm{C} 2, \mathrm{X})$, pour tout $X$;

(iv) $(\mathrm{C} 3, \mathrm{X})$, pour tout $X$ séparé. 
Remarques. (1) (C3,X) n'est pas nécéssairement vrai si $X$ n'est pas sépáre.

(2) Le théorème s'applique, en particulier, au cas où $K$ est répresentable.

Plus concrètement nous avons, par la Proposition 14

26. Proposition. Plaçons nous dans le Contexte II, et supposons que $K$ et $X$ sont deux objets Haussdorfs. Alors, les conditions suivantes sont équivalentes: (exC3, X) Pour tout ouvert $U \subset K \times X, \forall_{\pi} U$ est un ouvert dans $X$; $\left(C^{\prime} 3, X\right)$ Pour tout ouvert $U \subset \operatorname{Spec}(K \times X), \forall_{\pi} U$ est ouvent dans $\operatorname{Spec} X$ (i.e. la projection $\operatorname{Spec}(K \times X) \rightarrow \operatorname{Spec} X$ est fermée $)$.

D'où le théorème suivant:

27. THÉRÈME II (Théorème de caractérisation des compacts). E et $K$ étant placés dans les hypotheses du Théorème I. Alors $K$ est compact dans $\mathscr{E}$ selon la Définition 6 si et seulement si la projection $\operatorname{Spec}(K \times X) \rightarrow \operatorname{Spec}(X)$ est une application fermée, pour tout $X$ représentable.

\section{Exemples}

\section{En topologie}

Soit $\mathscr{C}$ la catégorie des espaces topologiques séparés, et $\mathscr{E}$ le topos des faisceaux sur $\mathscr{C}$ pour la topologie des recouvrements ouverts. Il est clair qu'on a la une exemple du Contexte II. Identifions $\mathscr{C}$ à une sous-catégorie pleine de $\mathscr{E}$. Alors, par construction de Spec on a Spec $X=X$ et $\operatorname{Spec}(X \times Y)=\operatorname{Spec} X \times \operatorname{Spec} Y$. Ceci montre que $X$ est séparé dans $\mathscr{E}$ si et seulement si il l'est au sens usuel. D'autre-part, il est bien connu que si $K$ est un espace topologique compact, alors les projections $\pi: K \times X \rightarrow X$ sont des applications fermées, quelque soit l'espace $X$. Réciproquement, soit $K$ un espace topologique quelconque, et $\mathscr{F}$ un ultrafiltre non principal sur $K$. Considérons alors $X=K \cup\{\infty\}$ muni de la topologie qui est discrete sur $K$ et pour laquelle les voisinages de $\infty$ son les ensembles appartenant à $\mathscr{F}$. Si $p \in K,\{p\} \notin \mathscr{F}$ (puisque $\mathscr{F}$ est nonprincipal) donc $K-\{p\}$ $\in \mathscr{F}$. Il s'en suit que $X$ est un espace séparé. Supposons maintenant que la projection $\pi: K \times X \rightarrow X$ soit une application fermée. Considérons alors $\Delta=$ $\{(p, p) / p \in K\}, \Delta \subset K \times X$, et soit $\bar{\Delta}$ la fermeture de $\Delta$ dans $K \times X$. Comme $K \subset \pi(\bar{\Delta}) \subset X$, et $\bar{K}=X$, il s'en suit que $\pi(\bar{\Delta})=X$. Donc $\infty \in \pi(\bar{\Delta})$, il existe alors $p_{0} \in K$ tel que $\left(p_{0}, \infty\right) \in \bar{\Delta}$. Si $V$ est un voisinage de $p_{0}$ et $F \in \mathscr{F}$ on a donc que $(V \times F) \cap \Delta \neq \varnothing$. C'est-a-dire, $V \cap F \neq \varnothing$. Ceci montre que, quelque soit $V$, voisinage de $p_{0}, V$ appartient à $\mathscr{F}$. C'est-à-dire, $\mathscr{F}$ converge vers $p_{0}$. On a donc démontré qu'un espace topologique $K$ est compact (au sens usuel) si et seulement si les projections $K \times X \rightarrow X$ vers tout espace séparé $X$, sont fermées. Donc, d'aprés le Théorème II on peut énoncer la proposition suivante. 
Un espace topologique $K$, séparé, est compact au sense usuel si et seulement si il est compact dans $\mathscr{E}$ au sense de la Définition 6.

2. En géométrie différentielle

(a) Soit $\mathscr{C}$ la catégorie des ouverts des espaces euclidiens $\mathbf{R}^{n}, n=0,1,2, \ldots$, et fonctions (indéfiniment) différentiables, et soit $\mathscr{E}$ le topos de faisceaux sur $\mathscr{C}$ pour la topologie des recouvrements ouverts. Il est clair qu'on a la un exemple du Contexte II. Toute variété indéfiniment différentiable $M$ définie un objet de $\mathscr{E}$ de la façon évidente. Une section $U \rightarrow M$ est simplement une application indéfiniment différentiable de $U$ dans $M$. Il est clair que cette objet est localement représentable. En effet, soit $\left(U_{\alpha} \hookrightarrow M\right)$ un recouvrement ouvert de $M$ par des ouverts euclidiens (au sens usuel), et soit $U \stackrel{x}{\rightarrow} M$ une section quelconque du faisceau $M$. Alors $x^{-1} U_{\alpha} \rightarrow U$ est un recourvrement de $U$ dans se site, et les restrictions $x^{-1} U \hookrightarrow U_{\alpha}$ son des sections du faisceau représentable $U_{\alpha}$. Ce qui montre que $\left(U_{\alpha} \hookrightarrow M\right)$ est une famille épimorphe dans $\mathscr{E}$. L'espace $\operatorname{Spec}(M)$ est l'espace topologique sous-jacent a la variété $M$, et on a $\operatorname{Spec}(M \times N)=\operatorname{Spec} M$ $\times \operatorname{Spec} N$. Donc $M$ est séparé dans $\mathscr{E}$ si et seulement si elle l'est au sens usuel. $\mathrm{Si}$ $M$ est compacte, alors les projections $M \times U \rightarrow U$ sont des applications fermées, pour tout espace $U$ du site. Réciproquement, soit $M$ une variété indéfiniment différentiable paracompacte, et soit $\left(S_{n}\right)$ une suite quelconque de $M$.

Supposons que la projection $M \times U \rightarrow U$ soit fermée pour tout espace $U$ du site. En particulier, la projection $\pi: M \times \mathbf{R} \rightarrow \mathbf{R}$ est fermée. Considérons alors $\Delta=\left\{\left(S_{n}, 1 / n\right): n \in \mathbf{N}\right\} \subset M \times \mathbf{R}$, et soit $\bar{\Delta}$ la fermeture de $\Delta$ dans $M \times \mathbf{R}$. Comme $0 \in \pi(\bar{\Delta})$, il existe donc $p_{0} \in M$ tel que $\left(p_{0}, 0\right) \in \bar{\Delta}$. Cela signifie qu'il existe une sous-suite de $\left(S_{n}\right)$ convergent vers $p_{0}$. Comme les suites suffisent pour caractériser la topologie des variétés paracompactes, on a démontré que $M$ est compact au sens usuel. Donc, d'aprés le Théorème II, on peut éncocer la proposition suivante.

Une variété indéfiniment différentiable (séparé et paracompacte) est compacte au sens usuel si et seulement si elle est compact dans $\mathscr{E}$ au sens de la Définition 6.

RemarQue. La ligne longue (voir [10] ou [1]) est une variété non paracompacte (et donc non compacte au sens habituel) qui est pourtant compacte dans $\mathscr{E}$ au sense de la Définition 6. On laisse au lecteur interessé le soin de le vérifier.

(b) Tout modele bien adapté $\mathscr{E}$ de la géométrie différentielle synthétique construit selon les techniques développées dans [3] se trouve dans le Contexte II. Pour fixer les idées, soit $\mathscr{E}$ le topos de faisceaux, pour la topologie des recouvrements ouverts, sur la catégorie $\mathscr{C}^{\text {op }}$, duals de celle des anneaux $C^{\infty}$ de la forme $A=C^{\infty}\left(\mathbf{R}^{n}\right) / I$, ou $I$ est un idéal de nature locale. C'est-à-dire; pour tout $f \in C^{\infty}\left(\mathbf{R}^{n}\right), f \in I$ si et seulement si, pour tout $x \in \mathbf{R}^{n},\left.\left.f\right|_{x} \in I\right|_{x}$, ou $\left.f\right|_{x}$ désigne le germe de $f$ en $x$, et où $\left.I\right|_{x}$ est l'idéal engendré, dans l'anneau des germes, par les 
éléments de la forme $\left.h\right|_{x}$ avec $h \in I$. Dans [3] on démontre que $\mathscr{E}^{\text {op }}$ est une catégorie d'espaces annelés et que les conditions du Contexte II sont vérifiées. Dans une autre optique, on peut définit $\mathscr{E}$ comme étant le topos des faisceaux sur $\mathscr{C}^{\mathrm{op}}$ pour la topologie engendrée par des familles de la forme $\left(A \rightarrow A\left\{a_{\alpha}^{-1}\right\}\right)$, où $a_{\alpha} \in A$ est une famille quelconque d'éléments de $A$ telle que $1 \in\left(a_{\alpha}\right)$. Précisons que $A\left\{A_{\alpha}^{-1}\right\}$ est la solution, dans $\mathscr{C}$, du problème universel que consiste a rendre inversible l'élément $a_{\alpha}$, et ( $\left.a_{\alpha}\right)$ est l'idéal de nature locale engendré par les $a_{\alpha}$. Des résultats démontrés dans [2] montrent que $\mathscr{C}$ est un exemple du Contexte II (voir aussi [6]). Si $A \in \mathscr{C}$, alors $\operatorname{Spec}(A)$ est l'ensembles $Z I \subset \mathbf{R}^{n}$ des zéros de l'ideál $I$ avec la topologie induite par celle de $\mathbf{R}^{n}$. Les objets $X$ localement représentables séparés sont éxactement les schémas $C^{\infty}$ séparés et $\operatorname{Spec}(X \times Y)=\operatorname{Spec} X \times$ Spec $Y$. Considérons maintenant l'objet $C^{\infty}(\mathbf{R})$ de $\mathscr{C}$. Alors, $\operatorname{Spec}\left(C^{\infty}(\mathbf{R})\right)=\mathbf{R}$ et comme dans a) on démontre que: $\mathrm{Si}$ les suites suffisent a caractériser la topologie de $\operatorname{Spec} X$, alors Spec $X$ est compact, au sens usuel, si et seulement si les projections sur tout objet du site sont fermées. En particulier, on a les énoncés suivants.

( $L$ 'objet dual d') un anneau $C^{\infty}, A=C^{\infty}\left({ }^{R n}\right) / I$, présenté par un idéal de nature local, est compact dans $\mathscr{E}$, au sens de la Définition 6, si et seulement si l'ensemble des zéros $Z I \subset \mathbf{R}^{n}$ de l'ideal I est compact au sens usuel.

Une variété indéfiniment différentiable (séparé et paracompacte) est compacte au sens usuel si et seulement si elle est compact $\mathscr{E}$ dans au sens de la Définition 6.

Remarquons que ce résultat est encore valable pour un schéma $C^{\infty}$ séparé quelconque tel que les suites caractérisent la topologie du spectre.

\section{En géométrie analytique}

Soit $\mathscr{C}$ la catégorie des modeles locaux pour les espaces analytiques, c'est-à-dire, les espaces anneles de la forme $O_{n}(U) / J$, où $O_{n}(U)$ est le faisceau des germes de fonctions holomorphes sur un ouvert $U \subset \mathbf{C}^{n}$, et $J$ est un sous-faisceau cohérent d'ideáux (voir par exemple Malgrange [7]). Soit $\mathscr{E}$ le topos des faisceaux sur $\mathscr{C}$ pour la topologie des recouvrements ouverts. Il est immédiat de constater que $\mathscr{E}$ est un exemple de Contexte II. Les objets $X \in \mathscr{E}$ localement représentables séparés sont éxactement les espaces analytiques que nous dénoterons $\left(X, O_{X}\right)$, où $X$ est l'espace topologique de base, et $O_{X}$ est le faisceau structurel. On a $\operatorname{Spec}\left(X, O_{X}\right)=X$, et $\operatorname{Spec}\left(\left(X, O_{X}\right) \times\left(Y, O_{Y}\right)\right)=\operatorname{Spec}\left(X, O_{X}\right) \times \operatorname{Spec}\left(Y, O_{Y}\right)$. Soit $\left(K, O_{K}\right)$ une espace analytique. Si $K$ est compact au sens usuel, les projections $K \times X \rightarrow X$ sont des applications fermées por tout espace $X$ et en particulier, pour tout modèle local $\left(X, O_{X}\right)$. Réciproquement, soit $\left(K, O_{K}\right)$ un espace analytique quelconque. Considérons le modele local $\left(X, O_{X}\right)=\left(\mathrm{C}, O_{\mathrm{C}}\right)$, c'est-àdire le corps des complexes avec le faisceau des germes de fonctions holomorphes, et supposons que la projection $K \times \mathbf{C} \rightarrow \mathbf{C}$ soit une application fermée. Comme dans l'Exemple 2(a), on en déduit que $K$ est compact au sens usuel. Donc, d'aprés le Théorème II, on peut énoncer la proposition suivante. 
Un espace analytique $\left(K, O_{K}\right)$ est compact au sens usuel si et seulement si il est compact dans $\mathscr{E}$ au sens de la Définition 6.

4. En géométrie algébrique

Soit $k$ un corps algébriquement clos, et soit $\mathscr{C}^{\mathrm{op}}$ la catégorie des $k$-algèbres de présentation finie. Soit $\mathscr{E}$ le topos des faisceaux sur $\mathscr{C}$ pour la topologie engendrée par les familles de la forme $A \rightarrow A\left[a_{i}^{-1}\right]$, où $a_{i} \in A, i=1,2, \ldots, n$, et $1 \in$ $\left(a_{1}, \ldots, a_{n}\right)$-c'est-à-dire, le "gros" topos de Zariski sur $k$ (voir [4]). Des résultats bien connus en géométrie algébrique montrent que $\mathscr{E}$ est un exemple du Contexte II dans lequel tout objet du site est séparé. $\mathrm{Si} A$ est une $k$-algèbre, considérée comme un objet de $\mathscr{E}$, alors $\operatorname{Spec} A$ est le spectre maximal de l'anneau $A$. En plus, le objets localement représentables sont exactement les $k$-schémas de présentation finie, et les objets séparés et localement représentables sont exactement les $k$-schémas séparés de présentation finie. Donc, d'aprés le Théoreme II, on peut énoncer la proposition suivante.

Un k-schéma séparé est complet au sens usuel si et seulement si il est compact dans $\mathscr{E}$ au sens de la Définition 6.

\section{References}

[1] E. J. Dubuc, 'Sur les modeles de la géométrie différentielle synthétique', Cahiers Topologie Géom. Différentielle Vol. XX-3, (1979).

[2] E. J. Dubuc, 'Open covers and infinitary operations in $C^{\infty}$-rings', Cahiers Topologie Géom. Différentielle, Vol. XXII-3, (1981).

[3] E. J. Dubuc, ' $C^{\infty}$-schemes', Amer. J. Math. 103 (1981), 683-690.

[4] M. Hakim, 'Topos annalés et schémas relatif', Ergeb. Math. Grenzgeb., Band 64 (Springer-Verlag, Berlin, Heidelberg, New York, 1972).

[5] A. Kock, 'Universal projective geometry; via topos theory', J. Pure Appl. Algebra 9 (1976), $1-24$.

[6] A. Kock, Synthetic differential geometry (Cambridge University Press, Cambridge, England, 1981).

[7] B. Malgrange, Analytic spaces (Monographie $\mathrm{N}^{\circ} 17$ de l'Enseignement Mathématique, Genève 1968).

[8] J. Penon, 'Topologie et intuitionnisme', dans Journées Faisceaux et Logique, Mai 1981 (Université Paris-Nord, pré-publications Mathématique (1982)).

[9] J. Penon, Manuscrits non-publiés, a paraitre dans la these de doctorat, Paris VII.

[10] M. Spivak, 'A comprehensive introduction to differential geometry, Vol. 1', Publish or Perish (Brandeis University, 1970).

Departamento de Matematicas

Universidad de Buenos Aires

1428 Buenos Aires, Argentina
U.E.R. de Mathématiques

Université de Paris VII

Tours 4555, 2 Place Jussieu

75005 Paris, France 